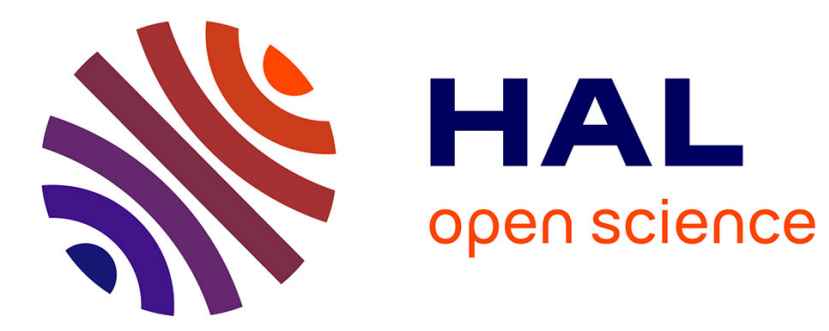

\title{
Resilient IoT-based Monitoring System for Crude Oil Pipelines
}

\author{
Safuriyawu Ahmed, Frédéric Le Mouël, Nicolas Stouls
}

\section{To cite this version:}

Safuriyawu Ahmed, Frédéric Le Mouël, Nicolas Stouls. Resilient IoT-based Monitoring System for Crude Oil Pipelines. Proceedings of the 7th International Conference on Internet of Things: Systems, Management and Security (IOTSMS 2020), Dec 2020, Paris, France. hal-03010657

\section{HAL Id: hal-03010657 https://hal.inria.fr/hal-03010657}

Submitted on 17 Nov 2020

HAL is a multi-disciplinary open access archive for the deposit and dissemination of scientific research documents, whether they are published or not. The documents may come from teaching and research institutions in France or abroad, or from public or private research centers.
L'archive ouverte pluridisciplinaire HAL, est destinée au dépôt et à la diffusion de documents scientifiques de niveau recherche, publiés ou non, émanant des établissements d'enseignement et de recherche français ou étrangers, des laboratoires publics ou privés. 


\section{Resilient IoT-based Monitoring System for Crude Oil Pipelines}

\author{
Safuriyawu Ahmed \\ University of Lyon, INSA Lyon, \\ CITI Lab \\ F-69621 Villeurbanne, France \\ Email: safuriyawu.ahmed@insa-lyon.fr
}

\author{
Frédéric Le Mouël \\ University of Lyon, INSA Lyon, \\ CITI Lab \\ F-69621 Villeurbanne, France \\ Email: frederic.le-mouel@insa-lyon.fr
}

\author{
Nicolas Stouls \\ University of Lyon, INSA Lyon, \\ CITI Lab \\ F-69621 Villeurbanne, France \\ Email: nicolas.stouls@insa-lyon.fr
}

\begin{abstract}
Pipeline networks dominate the oil and gas midstream sector, and although the safest means of transportation for oil and gas products, they are susceptible to failures. These failures are due to manufacturing defects, environmental effects, material degradation, or third party interference through sabotage and vandalism. Internet of Things (IoT)-based solutions are promising to address these by monitoring and predicting failures. However, some challenges remain in the deployment of industrial IoT-based solutions, as the reliability, the robustness, the maintainability, the scalability, the energy consumption, etc. This paper is therefore aimed at highlighting potential solutions for detection and mitigation of pipeline failures while addressing the robustness, the cost and scalability issues of such approach efficiently across the network infrastructure, data and service layers.
\end{abstract}

\section{INTRODUCTION}

Several incidents like nodes and pipeline vandalisation, corrosion, or leakages have been attributed to pipeline transportation failures both in developing and developed countries. Vandalisation or third party interferences are the leading cause of failures in developing countries. These failures have led to an annual loss of up to 10 billion USD in the United States [1]. Other effects include environmental disasters [2] and fatal incidents, one of which recorded deaths of over a thousand people in Jesse in the year 1998 [3].

Consequently, various systems are deployed to monitor pipeline infrastructures to reduce and mitigate failures. Current monitoring systems include daily overflights using stateof-the-art high-definition cameras by specialised helicopters, drones, community and security-based surveillances - all largely dependent on humans, all truly expensive and not realtime-based. Alternative wired systems using fibre optics or copper are used, but are limited by high installation and maintenance costs, difficulty in fault location and repairs, inflexibility to harsh terrain and the possibility to be compromised through third party interference [4]. Other monitoring systems include the use of the supervisory control and data acquisition system (SCADA) by the computational analysis of their pressure, volume and temperature through the deployment of sensor and communication networks [5]. Despite a popular legacy monitoring system, SCADA is characterised by its high cost, long delays, and inflexibility to protocol changes attributes [4], [6], [7], which are in contrary to the fundamental requirements of monitoring systems from pipeline operators, i.e. sensitivity, accuracy, and cost [8]. The inefficiency of the aforementioned monitoring systems is further ascertained with the recorded loss of crude oil from the pipeline network of Shell Nigeria to the tune of 11000 barrels per day in 2018 due to failures, an increment of nearly $550 \%$ compared to the previous year [9]. It is therefore paramount to have in place a system which can monitor - in real-time - operations of the pipeline to determine in a timely manner any anomaly that occurs.

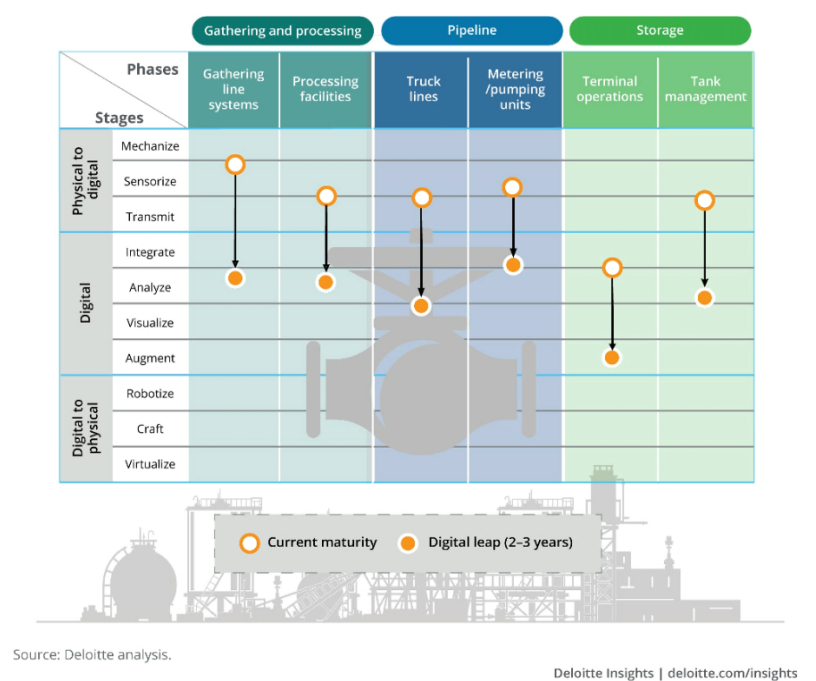

Fig. 1. Digital Maturity of Midstream Operations [5]

More recent approaches to monitor pipeline networks are based on wireless sensor networks (WSN) and Internet of Things (IoT). Such approaches are shown to be more efficient [10]-[14]. They rely on metrics such as energy consumption, reliability, scalability for long-haul networks [4] and robustness - to failures and third-party interference. These constraints affect their cost of implementation, resilience and maintenance. As a consequence, it limits their adoption by oil and gas companies. Fig. 1, extracted from [5], shows the current state of the digital maturity of the midstream sector. It shows that despite the numerous research, adoption is still an early stage hence the relevance of this work. 
In this paper, we present a preliminary work on an end-toend resilient IoT-based monitoring system for a fault-tolerant, accurate and real-time monitoring of crude oil pipelines. We propose a distributed placement and estimation for leakage detection and localisation while considering cost, scalability and efficiency as a trade-off. The remainder of this work is structured as follows: in section II, related work will be discussed, followed by our research plan in section III and our first simulations in section IV. In section $\mathrm{V}$, we conclude and present future works.

\section{RELATED WORK}

Recent research efforts to develop more efficient WSNand IoT-based infrastructure monitoring systems vary from the architectural design, sensor placements, algorithms to data and service management. The following subsections present research works in these areas.

\section{A. IoT/WSN for various applications}

A four-layered architectural framework covering the sensor layer to the cloud servers for low power IoT applications was proposed by Yelmarthi et al. [15]. It is a hybrid of wired and WSN, which is said to be easily implemented with minimal resources for diverse application range. Experiments were carried out on posture and physical activities, damage detection and power consumption to show its applicability in multiple industries and prove its low power consumption. [16] considered generic sensor placement for the optimisation of the sensor network using both discrete power control and retransmission for one and two-tier uniformly and non uniformly distributed WSN. Boubrima et al. [17] worked on optimal sensor deployment to ensure minimal cost for air pollution monitoring. The cost was minimised by formulating the problem using real air pollution dispersion, network coverage, and connectivity constraints. Their approach, which has a centralised architecture, showed a considerable reduction in cost. Jamali-Rad et al. [18], in their work, examined the feasibility of using IoT-based Low Power Wide Area Networks (LPWAN) for seismic quality control. They tested LoRaWAN by observing the packet loss rate over a single link and small-scaled network and its performance in the presence of interference and mobility. These tests show that LoRa performs considerably well with interferences from UMTS LTE and GSM close to the gateway and also against the doppler effect. Rudes et al. [19] tested, on a small-scale basis, the reliability of the LoRaWAN communication protocol for IoT applications. They conducted the test by working on varying parameters such as packet size, distance, and terrain for precision agriculture and wildfire detection. Despite the lack of optical visibility between some of the nodes and the central station, the results obtained were promising.

Thus far, we have discussed works focused mainly on general guidelines for deploying IoT based applications. It was shown that network coverage, communication protocol, connectivity, architectural designs, and the propagation model of the monitored quantity affect performance and cost. Proposed archi- tectures are, however, centralised making them susceptible to Single Points of Failure (SPOF).

\section{B. IoT/WSN for pipelines}

Khan et al. [6] in their work proposed an IoT architecture for the three sectors of the oil and gas industry. They took into consideration in each layer, the design aspect that will guarantee reliability and robustness through hierarchical architecture by recommending a three-layered architecture. It was structured to interconnect and collaborate to enhance performance through intelligent decision makings and reliable communication while allowing predictive maintenance. Various processes of using WSN for monitoring pipeline and mitigating vandalism were shown in [12], [20]-[22]. In their work, they highlighted the benefits and challenges of using this approach in comparison with other methods. Yu et al. [13] proposed a three-layered architecture and an information collection WSN algorithm using sensor nodes that are capable of continuously detecting hydraulic pressure flow, quantity oil and gas leak corrosions in the pipeline. Network optimisation was done through data delivery algorithm, data fusion and line deployment strategy. These strategies were expected to reduce delay for pre-prioritised events, reduce redundancy and avoid unbalanced energy consumption. The algorithm is not scalable with the proposed line deployment strategy as nodes will exponentially increase as the distance to the server decreases. [23] also worked on node placement in a linear oil pipeline for maximal lifetime operations. They used an equal distance scheme with a realistic power distance model defined by the length of the pipeline segment divided by the maximum transmission range of the sensor node in which case we can have the lowest number of sensors deployed. This method may significantly increase the energy consumption of the nodes. Other methods of determining sensor placement include the work of Perelman et al. [24]. They proposed a minimum test cover approach for fault detection in pipelines using an approximate solution of the minimum set cover problem. Sela et al. [25] also worked on robust placement of sensors in a pipeline network. They proposed robust mixedinteger optimisation (RMIO) and robust greedy approximation (RGA) which were enhancement to nominal MIO and GA using a redundancy and robustness parameter. In most cases, RMIO and MIO outperformed the robust sub-modular function optimisation (RSFO), MIO and GA versions in a test conducted through the simulation of real water networks. While the works above have demonstrated the feasibility of their approach, they did not put into consideration the resiliency of the system, especially in long-distance pipelines. They also assume that a single sensor can detect failures in multiple pipelines.

\section{Data and Service Management}

The operations of the oil and gas industries present a huge amount of data [26]. In the midstream sector alone, every 150,000 miles of pipeline produces up to 10 terabytes of 
data [1]. While several efforts have been made to gain insights into these data in the upstream and downstream sectors [27], [28] through big data analytics, the midstream sector is left almost unexploited. Efficient data and service management in the midstream sector can reduce the annual downtime by $70 \%$ and the associated cost by $22 \%$ [29] through timely failure detection and predictive maintenance. Other driving factors for pipeline data management consist of ageing infrastructure and outdated technology, incessant vandalisation, and increased vulnerability to cyberattacks [29], [30]. Shoja et al. [7] illustrated how IoT in the oil and gas industry could be used to implement a simple, secure, quick, and reliable data collection. [26], [28] also show the benefits and importance of data management and analytics in this context. Other works that can be applied to pipeline data and service management are as follows: [31]-[33] proposed a data placement strategy for fog infrastructure aimed to reduce the overall network or service latency of data storage and retrieval. [34], [35] worked on middleware architecture and services to ensure systems robustness. [36] considered fault management and tolerance services, recovery, and restoration services using the self-healing through structural adaptation (SHSA) algorithm. Authors in [37] define an IoT service placement architecture that incorporates cloud and fog computing taking into consideration latency and system's condition and implements a service orchestrator algorithm for the architecture. The proposed architecture takes advantage of the fog environment by placing services according to the state of the network and the user's and servers' location.

Authors in [38] proposed an optimal data scheduling policy using multiple channels to minimise real-time delay through distributed and 4-layer fog computing architecture.

To the best of our knowledge, no work has considered placement and distributed leakage estimation as a fault-tolerant and robust approach to mitigating failures in crude oil pipelines across multiple layers.

\section{Contribution AND Research Plan}

In this work, we propose a hierarchical and distributed network architecture for the implementation of the pipeline monitoring system. This kind of architecture prevents single points of failure (SPOF) usually associated with centralised systems. Our goal is to allow for a scalable network without substantially affecting performance metrics such as accuracy of the leakage detection and localisation, latency, cost, and energy consumption. As shown in Fig. 2, the proposed network architecture is based on a 3-tier architecture. Layer 1, consists of the sensors connecting to other sensors, one or multiple gateways. Geographically close sensors will share information to take advantage of the spatial correlation of data allowing distributed detection and localisation. In Layer 2, we take advantage of the fog paradigm to reduce latency and energy consumption for more complex computations in the gateways. Layer 3 is implemented for long-term storage and historical analysis of data and services. In terms of communication, we make use of LoRaWAN for both the short and long-range communications. We also propose the use of a cellular network as a backhaul as they are more widely deployed. Based on

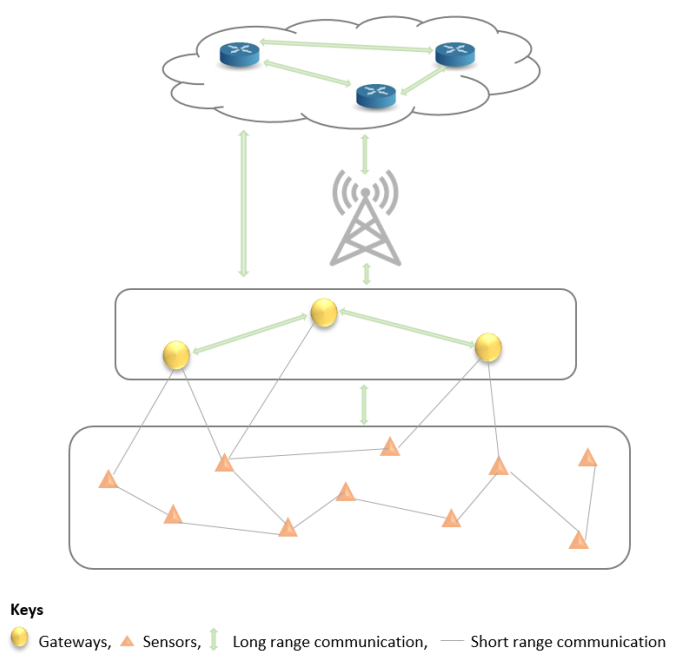

Fig. 2. Proposed Network Architecture

Fig. 2, this paper introduces our work on Placement and Distributed Estimation for Leakage Detection and Localisation on Oil Pipelines, which is divided into two parts: a) Node placement, distributed detection and localisation b) Distributed data and service management. We consider as a tradeoff the accuracy of leakage detection and localisation, the cost, the fault-tolerance, the scalability, and the efficiency of the system in terms of data storage, packets transmitted and energy consumption.

\section{A. Node placement, distributed detection and localisation}

The efficiency of any monitoring system can be largely influenced by the coverage of the monitored event, the detection technique and the overall resilience of the system to failures (communication, node, etc.). Therefore, for our node placement, we put the aforementioned factors into account as explained later in this subsection. In this work, we concentrate only on existing long-haul transmission pipeline networks for better representation of the industrial reality.

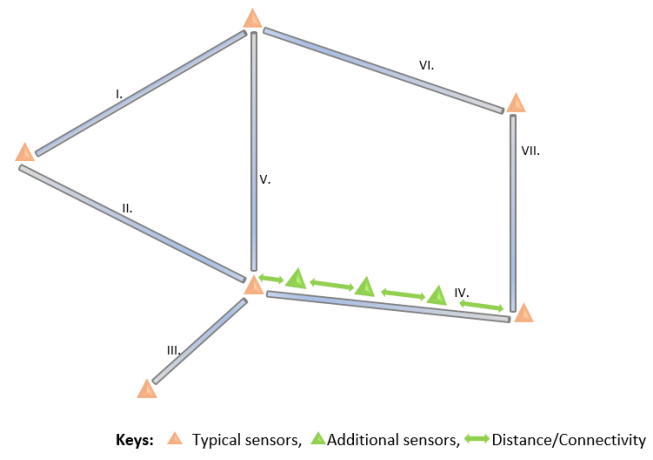

Fig. 3. Sensor placement on long transmission crude oil pipelines 
1) Coverage: New pipelines are sometimes equipped with sensors, but only at the key junctions [1] as we have illustrated in Fig. 3 using the typical sensors. This method of sensor placement may not allow detection of small leaks in transmission lines and are also not resilient to failures like sensor vandalism, hardware or communication failure. Other node placement techniques include taking into consideration, the maximum communication range of the sensors [23] or the shortest distance between monitored events as in [24], [25]. However, leakage events are stochastic in nature hence this distance cannot be predetermined. Therefore, to ensure maximum event coverage, we propose sensor deployment that is based on the crude oil propagation properties in a pipeline and the communication range of the sensors. This is to ensure a nearly optimal event coverage as demonstrated in [17] where sensor placement for detecting air pollution was optimised using the Gaussian dispersion of the pollutant. Our work differs from theirs however, as we use the pressure wave generated due to leakage and the resulting changes in the pressure gradient of the flow. With the increment in density as a result of such placement the resilience to failure can be improved as a result of the additional sensor(s) that is able to detect leakage. Hence if a sensor is failing, detection or localisation will still be possible using the other ones as shown on pipeline $I V$ in Fig 3.

However, we also aim to find an optimal distance between sensors that allows detection of small-sized to big-sized leaks. To achieve that, we consider the amplitude of the pressure wave whose value is dependent on the size of the leak and attenuates with distance. Therefore, the distance constraint is defined such that it is less than the maximum detecting distance for flows in a steady state. This can be calculated with the attenuation model for sound wave where the maximum distance is the longest distance from the leak point at which the wave is still detectable. The density of nodes deployed within this bound can include some level of redundancy to ensure a trade-off between detectability and failure.

2) Detection Technique: Traditional monitoring system are characterised by inefficiency and a high false-alarm rate [14]. Although [39]-[41] highlights several techniques for leakage detection in pipelines, some of these techniques are computationally complex [8] and even more challenging with resource constraints, i.e. memory, energy consumption, limited bandwidth/delayed networks [4] such as in WSN which are also highly prone to failures [42]. Other challenges include a centralised approach of the previous works, making them susceptible to SPOF. Thus, in our work, we consider the implementation of leakage detection and localisation by first ensuring that the sensor nodes are in a reliable state, i.e. a considerable number of nodes are behaving as expected. Then, we make use of the combination of several leakage detection methods, i.e. pressure point analysis (PPA), the gradientbased (GM) and the negative pressure wave method (NPWM) in a distributed manner. These methods are software-based non-invasive methods that are less computationally complex compared to others [8], making them suitable for WSN and easy to deploy on existing pipeline networks. However, the proposed detection methods have their individual strengths and weaknesses e.g. the ease of detection of leakages in transient or steady states, the accuracy of localisation etc. (details can be found in [8], [41]). We therefore study the fusion of these three methods by taking advantage of their strengths to improve the accuracy of detection and reduce or eliminate false positive through asynchronous consensus. To implement, we follow these steps:

(i) Ensure that more than half the number of detecting sensors are not faulty.

(ii) For a detection to be valid, at least two of the implemented techniques must detect leakage.

(iii) Find the leakage region i.e. the region where the negative pressure wave (NPW) or the change in gradient is detectable.

(iv) Localise the leakage using only sensors in this region in a distributed manner.

Contrary to the existing solutions where all information is collected at the gateway to detect and localise leakages, our proposed detection technique will make use of partial information from a defined set of neighbouring sensors as described in III-A1. With the limited data, we aim to maintain or improve the accuracy of the localisation and reduce false positive in detection. Thus, a performance evaluation will be conducted to compare our technique and the centralised versions (NPWM and GM). For such evaluation, we need to define a quantified criterion expressing the detection and localisation accuracy of a leakage. Hence, we first define the number of sensors $n_{c}$ for the classical approach and the number of sensors $n_{p}$ for our proposed approach as described above. Let $e_{n_{c}}$ denote the error in leakage location and detection using $n_{c}$ sensors and $e_{n_{p}}$ using $n_{p}$ sensors. Our goal is to minimise the objective function $E$ defined as

$$
E=\left(e_{n_{c}}-e_{n_{p}}\right)
$$

where $E=$ the error margin between the classical approach and our proposed approach.

3) Resilience: One of the downsides of distributed systems is the communication overhead and energy consumption resulting from the mesh connection of the nodes. Having a fullymeshed connection makes scalability expensive but provides the best resilience. We will like to minimise the number of connections among the nodes while still ensuring resiliency to link failure. Hence, we propose a generalised connectivity constraints to be implemented using an adjacency matrix which is dependent on the topology of the pipeline network for communication among the nodes. Such matrix will be subject to minimising the objective function $(\mathrm{O})$ i.e. the total number of connections among the nodes. These constraints include the ability to communicate with an upstream neighbour, a down stream neighbour and a direct or indirect connection with at least one gateway. Also, each node should be within distance constraint explained in subsection III-A1. 
Therefore (1) can be modified as

$$
E_{O}=\left(e_{n_{c}}-\bar{e}_{n_{p}}\right)
$$

where $\bar{e}_{n_{p}}$ is the error obtained using $e_{n_{p}}$ subject to the objective function and $E_{O}$ equals the error margin.

\section{B. Data and Service Management}

As shown in Fig. 2, we proposed a fog-enabled architecture that allows the placement of data and services closer to the user. This is a common requirement for IoT systems to reduce latency as well as energy consumption. Still in existence however, are some challenges as follows:

1) Increment in latency due to data misplacements in fogenabled architectures [33].

2) Data loss as a result of node failure.

3) Increase in energy consumption due to multi-hop communication or need for spatial or time correlation of data in distributed systems.

4) Constrained resources i.e storage capacity of nodes.

As such, adequate data and service management becomes imperative. Thus, we propose such management as an optimisation problem through proper placement, replication and migration of data and services using a game theory approach. The aim is to improve the availability, fault tolerance and scalability of the system while considering energy consumption, latency and the storage capacity of the nodes as constraints.

Let player 1 represent the pipeline monitoring system and player 2 the failure elements. We want to guarantee a certain level of performance $(Z)$ for the monitoring system $M$ (player 1 ) in the event of unknown node failures or interference $F$ (player 2). $Z$ can be defined as the value function to estimate the performance of $M$ where

$$
\underline{Z_{M}}=\max _{M} \min _{F} Z_{M}\left(A_{M}, A_{F}\right)
$$

We have two sets of actions, $A_{M}$ and $A_{F}$ and two states, State $_{0}$ and State $_{1}$. The timeline $t_{0}$ and time $t_{1}\left(t_{1}>t_{0}\right)$ are for states $S_{t a t e_{0}}$ and $S_{\text {tate }}$ respectively.

$A_{M}=\left\{I_{d s}, R_{d s}, M_{d s}\right\}$ represents the set of possible actions of $M$ (player 1). $I_{d s}$ (initial data and service placement) denotes the first action taken by $M$ in State $_{0}$ using random nodes. $R_{d s}$ and $M_{d s}$ (data and service replication and migration respectively) denote the subsequent actions i.e in State 1 . Whereas $R_{d s}$ is an action taken to counter failures such as node failures, communication failures etc. $M_{d s}$ is specific to needs such as lack of storage capacity, increased delay, insufficient or non-utilisation of resources etc. All actions taken by $M$ will take into account the overall cost i.e. data access delay, energy consumption, cost of redirection etc.

$A_{F}=\left\{f_{1}, f_{2}, \ldots, f_{r}\right\}$ contains a set of potential actions of $F$ (player 2), where $f_{i}$ represents failures in the network, i.e. communication failures, packet loss, hardware failures etc. and $r$ equals the total number of possible actions. For $F$, we assume that all nodes are in a good state at $t_{0}$ hence no action taken in State $_{0}$. On the other hand, any sort of failure represents the possible action of $F$ in State $_{1}$.
For the first part of the research, we are currently simulating oil propagation and leakages in pipeline using NS3. This simulation is targeted towards obtaining results to determine the performance of the classical approach in an ideal condition. The next step is the implementation of our proposed distributed solution and the comparison of these two methods in both ideal and non-ideal conditions.

\section{PReliminary Results}

To evaluate the individual strengths (detection or localisation) of the methods mentioned above (NPWM, PPA and GM), we used NS3 to simulate crude oil propagation and leakages. This was done in a single horizontal transmission pipeline segment for a one-phase flow. We aim to determine node placement that allows efficient detection of small to large-sized leaks and to check the performance of NPWM and GM based on this placement. Table (I) shows the simulation parameters. In these first simulations, we tested NPWM and PPA for detection, and NPWM and GM for localisation. As a primary test, we have not included any sort of failure in terms of communication, node or infrastructure i.e. test are conducted in ideal conditions for each method. Leakage localisation is also done in a centralised manner using information from all the sensor nodes. The results from these simulations will allows us to carry out a comparative analysis of the centralised version to our distributed version in future works.

TABLE I

SIMULATION PARAMETERS

\begin{tabular}{|c|c|}
\hline Type & Transmission line \\
\hline Material & Carbon steel \\
\hline Length & $20 \mathrm{~km}$ \\
\hline Wall thickness & $0.323 \mathrm{~m}$ \\
\hline Inside diameter & $0.61 \mathrm{~m}$ \\
\hline Height/elevation & $0 \mathrm{~m}$ \\
\hline Oil kinetic viscosity & $2.90 \mathrm{~mm}^{2} / \mathrm{s}$ \\
\hline Temperature & $50^{\circ} \mathrm{C}$ \\
\hline Oil density $(\rho)$ & $837 \mathrm{~kg}^{3} / \mathrm{m}^{3}$ \\
\hline Inlet pressure $\left(P_{0}\right)$ & $1000 \mathrm{psi}$ \\
\hline Reynolds no $\left(R_{e}\right)$ & 1950 \\
\hline Velocity $(U)$ & $2 \mathrm{~m} / \mathrm{s}$ \\
\hline Oil modulus elasticity $(K)$ & $1.85 * 10^{5} \mathrm{psi}$ \\
\hline Carbon steel modulus elasticity $(e)$ & $3 * 10^{6} \mathrm{psi}$ \\
\hline Gravititational force $(\mathrm{g})$ & $9.81 \mathrm{~m} / \mathrm{s}^{2}$ \\
\hline Constant $(e)$ & 2.718 \\
\hline Wave speed $(c)$ & $14.1 \mathrm{~m} / \mathrm{s}$ \\
\hline Coefficient of friction $(\lambda)$ & 0.033 \\
\hline Packet size & $32 \mathrm{bytes}$ \\
\hline Data rate & $1 \mathrm{Kbps}$ \\
\hline
\end{tabular}

\section{A. Node Placement and Detection}

We examine the impact of several amplitudes on the detectability of leakages using the NPWM. We tested $0.5 \mathrm{kpsi}$, $5 k p s i$ and $20 k p s i$ at various distances between sensors for small, medium and large leaks. While the 20kpsi is detectable at a distance of less than $2500 \mathrm{~m}$ between sensors, the $0.5 \mathrm{kpsi}$ amplitude is detectable at up to approximately $1500 \mathrm{~m}$. Figure (4) shows the result of this test. 


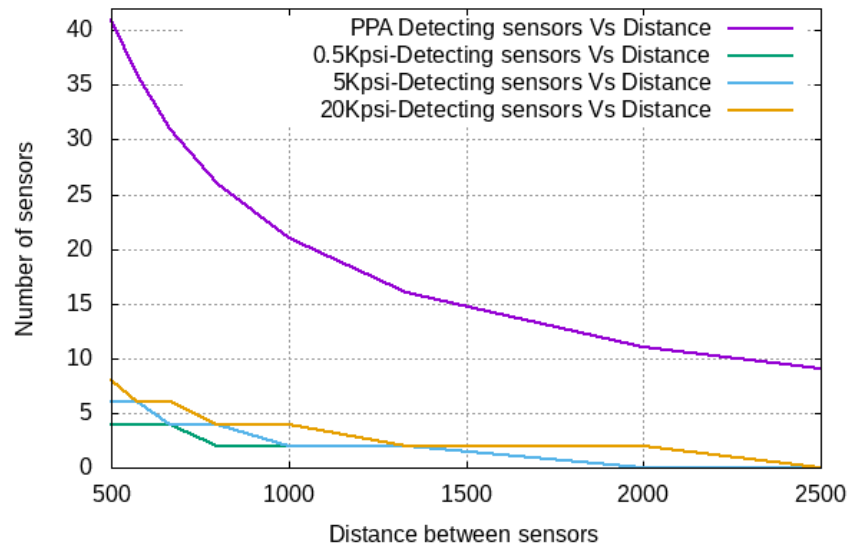

Fig. 4. NPWM and PPA

The PPA method detectability is based on the leakage size. With a detectable leak size, all sensor nodes along the pipeline can eventually detect the leakage based on the pre-estimated pressure and a pre-defined threshold value. With these results, we identified the number of sensors to be placed on the pipeline and the distance between them to make small to bigsized leakages detectable. Fig (4) shows that the NPWM is not very efficient in detecting leakages from a long-range. Hence we selected an average distance of $1000 \mathrm{~m}$ where all three amplitudes are detectable and also allows a gradient-based detection.

\section{B. Localisation}

The leakage localisation test was conducted by randomly selecting a sample of leak points across the pipeline. The aim of this test is to determine the accuracy of localisation using the centralised NPWM and GM, which will later be compared to our proposed distributed version. With a confidence level of $98 \%$, the confidence interval of the sample data on which the test was conducted is approximately $9880 m-14523 m$. The localisation accuracy $\left(L_{A}\right)$ was calculated using the following equation:

$$
L_{A}=100-\left(\frac{a b s\left(C_{l}-A_{l}\right)}{A_{l}} * 100\right)
$$

where $C_{l}$ is the calculated leak location and $A_{l}$ is the actual leak location.

Additional simulation settings i.e. the number of nodes and the distance between them are based on the results from section IV-A. Other results from the simulation, as shown in Fig (5) shows that NPWM performed excellently well in terms of localisation compared to the GM. It shows a consistent localisation accuracy of over $99 \%$. GM performance on the other hand is not as consistent as that of the NPWM. However, it also has a very good localisation accuracy with an average of about $98 \%$.

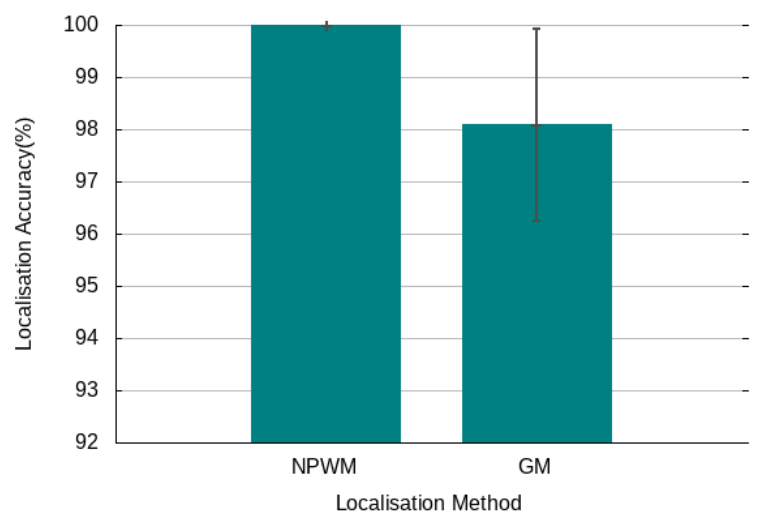

Fig. 5. NPWM and GM

\section{CONCLUSION AND FUTURE WORK}

In this paper, we have introduced our work on an IoTbased monitoring system for crude oil pipelines that ensures resiliency across the network infrastructure. We propose a system that is expected to be cost-efficient, scalable, and efficient by considering trade-offs between density, detectability and cost. An implementation through simulation is currently being carried out to evaluate the performance of the system. The preliminary results for the centralised detection/localisation show the performance of each method in detection and or localisation. To overcome the SPOF issue associated with this method, we will use data replication and asynchronous consensus across the nodes for a distributed decision. Besides, we intend to implement services for data filtering, categorisation, prioritisation, historical data computation for trends and emergencies. Further work will also be conducted in deploying the distributed monitoring system on a real pipeline network.

\section{ACKNOWLEDGMENT}

This work was supported by the Petroleum Technology Development Fund (PTDF).

\section{REFERENCES}

[1] A. Slaughter, G. Bean, and A. Mittal, "Connected barrels: Transforming oil and gas strategies with the Internet of Things," Deloitte Center for Energy Solutions, Tech. Rep., 2015.

[2] A. Ambituuni, J. Amezaga, and E. Emeseh, "Analysis of safety and environmental regulations for downstream petroleum industry operations in nigeria: Problems and prospects," Environmental Development, vol. Volume 9, p. Pages 43-60, 012014.

[3] A. Ambituuni, P. Hopkins, J. Amezaga, D. Werner, and J. Wood, Risk Assessment Of A Petroleum Product Pipeline In Nigeria: The Realities Of Managing Problems Of Theft/sabotage, ser. WIT Transactions on The Built Environment. WIT Press, 2015, vol. VI, pp. 49-50.

[4] M. Y. Aalsalem, W. Z. Khan, W. Gharibi, M. K. Khan, and Q. Arshad, "Wireless sensor networks in oil and gas industry: Recent advances, taxonomy, requirements, and open challenges," Journal of Network and Computer Applications, vol. 113, pp. 87-97, 2018.

[5] A. Slaughter, A. Mittal, and V. Bansal, "Bringing the digital revolution to midstream oil and gas," Tech. Rep., 2018.

[6] W. Z. Khan, M. Y. Aalsalem, M. K. Khan, M. S. Hossain, and M. Atiquzzaman, "A reliable Internet of Things based architecture for oil and gas industry," in 19th International Conference on Advanced Communication Technology (ICACT), Feb 2017, pp. 705-710. 
[7] S. Shoja and A. Jalali, "A study of the Internet of Things in the oil and gas industry," in 4th International Conference on Knowledge-Based Engineering and Innovation (KBEI), Dec 2017, pp. 0230-0236.

[8] P. Ostapkowicz, "Leak detection in liquid transmission pipelines using simplified pressure analysis techniques employing a minimum of standard and non-standard measuring devices," Engineering Structures, vol. 113, pp. 194-205, apr 2016.

[9] SPDCN, "Security, theft, sabotage and spills," Shell Petroleum Development Company of Nigeria Limited, Tech. Rep., 2017.

[10] J. Kim, G. Sharma, N. Boudriga, and S. S. Iyengar, "Spamms: A sensorbased pipeline autonomous monitoring and maintenance system," in 2010 Second International Conference on COMmunication Systems and NETworks (COMSNETS 2010), Jan 2010, pp. 1-10.

[11] A. M. Sadeghioon, N. Metje, D. N. Chapman, and C. J. Anthony, "Smartpipes: Smart wireless sensor networks for leak detection in water pipelines," Journal of Sensor and Actuator Networks, 2014.

[12] N. F. Henry and O. N. Henry, "Wireless sensor networks based pipeline vandalisation and oil spillage monitoring and detection: Main benefits for nigeria oil and gas sectors," The SIJ Transactions on Computer Science Engineering \& its Applications (CSEA), vol. 3, no. 1, Jan. 2015.

[13] H. Yu and M. Guo, "An efficient oil and gas pipeline monitoring systems based on wireless sensor networks," in International Conference on Information Security and Intelligent Control, Aug 2012, pp. 178-181.

[14] S. Rashid, U. Akram, S. Qaisar, S. A. Khan, and E. Felemban, "Wireless sensor network for distributed event detection based on machine learning," in 2014 IEEE International Conference on Internet of Things (iThings), and IEEE Green Computing and Communications (GreenCom) and IEEE Cyber, Physical and Social Computing (CPSCom), Sep. 2014, pp. 540-545.

[15] K. Yelmarthi, A. Abdelgawad, and A. Khattab, "An architectural framework for low-power IoT applications," in 2016 28th International Conference on Microelectronics (ICM), Dec 2016, pp. 373-376.

[16] R. Li, M. Wenting, N. Huang, and R. Kang, "Deployment-based lifetime optimization for linear wireless sensor networks considering both retransmission and discrete power control," PLOS ONE, vol. 12, 11 2017.

[17] A. Boubrima, F. Matigot, W. Bechkit, H. Rivano, and A. Ruas, "Optimal deployment of wireless sensor networks for air pollution monitoring," in 24th International Conference on Computer Communication and Networks (ICCCN), Aug 2015, pp. 1-7.

[18] H. Jamali-Rad, X. Campman, I. MacKay, W. Walk, M. Beker, J. van den Brand, H. Jan Bulten, and V. van Beveren, "IoT-based wireless seismic quality control," The Leading Edge, vol. 37, pp. 214-221, 032018.

[19] H. Rudeš, I. N. Kosović, T. Perković, and M. Čagalj, "Towards reliable IoT: Testing lora communication," in 2018 26th International Conference on Software, Telecommunications and Computer Networks (SoftCOM), Sep. 2018, pp. 1-3.

[20] F. C. Obodoeze, H. C. Inyiama, and V. Idigo, "Wireless sensor network in niger delta oil and gas field monitoring: The security challenges and countermeasures," International Journal of Distributed and Parallel Systems (IJDPS), vol. 3, no. 6, Nov. 2012.

[21] A. C. Azubogu, V. E. Idigo, S. U. Nnebe, O. S. Oguejiofor, and S. E., "Wireless sensor networks for long distance pipeline monitoring," International Journal of Electrical, Computer, Energetic, Electronic and Communication Engineering, vol. 7, no. 3, pp. 285 - 289, 2013.

[22] K. Yunana, S. O. Adewale, A. A. Alfa, and M. Sanjay, "An exploratory study of techniques for monitoring oil pipeline vandalism," Covenant Journal of Engineering Technology (CJET) Vol. 1, No. 1, June 2017, vol. 1, no. 1, Jun. 2017

[23] Y. Guo, F. Kong, D. Zhu, A. Saman Tosun, and Q. Deng, "Sensor placement for lifetime maximization in monitoring oil pipelines," Proceedings of the 1st ACM/IEEE International Conference on CyberPhysical Systems, ICCPS '10, 052010.

[24] L. S. Perelman, W. Abbas, X. Koutsoukos, and S. Amin, "Sensor placement for fault location identification in water networks: A minimun test approach," Automatica, vol. 72, pp. 166-176, Oct. 2016.

[25] L. Sela and S. Amin, "Robust sensor placement for pipeline monitoring: Mixed integer and greedy optimization," Advanced Enginnering Informatics, vol. 36, pp. 55-63, Apr. 2018.

[26] M. Hajirahimova, "Opportunities and challenges big data in oil and gas industry," 112015.

[27] M. Mohammadpoor and F. Torabi, "Big data analytics in oil and gas industry: An emerging trend," Petroleum, 122018.
[28] R. Aliguliyev and Y. Imamverdiyev, "Conceptual big data architecture for the oil and gas industry," Problems of Information Technology, vol. 08, pp. 3-13, 012017

[29] A. Mittal, A. Slaughter, and P. Zonneveld, "Bringing the digital revolution to midstream oil and gas," Deloitte Center for Energy Solutions, Tech. Rep., 2018.

[30] P. Zonneveld, A. Slaughter, and A. Mittal, "Protecting the connected barrels cybersecurity for upstream oil and gas," Deloitte Center for Energy Solutions, Tech. Rep., 2017.

[31] A. Aral and T. Ovatman, "A decentralized replica placement algorithm for edge computing," IEEE Transactions on Network and Service Management, vol. 15, no. 2, pp. 516-529, June 2018.

[32] Y. Shao, C. Li, and H. Tang, "A data replica placement strategy for IoT workflows in collaborative edge and cloud environments," Computer Networks, vol. 148, 112018.

[33] M. I. Naas, P. R. Parvedy, J. Boukhobza, and L. Lemarchand, "iFogStor: An IoT data placement strategy for fog infrastructure," in IEEE 1st International Conference on Fog and Edge Computing (ICFEC), May 2017, pp. 97-104.

[34] N. Mohamed, J. Al-Jaroodi, I. Jawhar, S. Lazarova-Molnar, and S. Mahmoud, "Smartcityware: A service-oriented middleware for cloud and fog enabled smart city services," IEEE Access, vol. 5, pp. 17576-17588, 2017.

[35] A. Giordano, G. Spezzano, and A. Vinci, "Smart agents and fog computing for smart city applications," in International Conference on Smart Cities, 06 2016, pp. 137-146.

[36] D. Ratasich, F. Khalid, F. Geissler, R. Grosu, M. Shafique, and E. Bartocci, "A roadmap toward the resilient Internet of Things for cyberphysical systems," IEEE Access, vol. 7, pp. 13 260-13 283, 2019.

[37] K. Velasquez, D. P. Abreu, M. Curado, and E. Monteiro, "Service placement for latency reduction in the internet of things," Annals of Telecommunications, vol. 72, pp. 105-115, Feb. 2017.

[38] W. Wang, G. Wu, Z. Guo, L. Qian, L. Ding, and F. Yang, "Data scheduling and resource optimization for fog computing architecture in industrial IoT," 15th International Conference, ICDCIT, pp. 141-149, 012019.

[39] R. Beushausen, S. Tornow, H. Borchers, K. Murphy, and D. J. Zhang, "Transient leak detection in crude oil pipelines," in International Pipeline Conference, 2004.

[40] U. Roy, "Leak detection in pipe networks using hybrid ann method," Water Conservation Science and Engineering, vol. 2, no. 4, p. 145-152, 2017.

[41] T. R. Sheltami, A. Bala, and E. M. Shakshuki, "Wireless sensor networks for leak detection in pipelines: a survey," Journal of Ambient Intelligence and Humanised Computing, vol. 7, pp. 347-356, Mar. 2016.

[42] K. P. Sharma and T. Sharma, "rdfd: reactive distributed fault detection in wireless sensor networks," Wireless Network, Feb. 2016. 